\title{
Three Dimensional Adaptive Method for Compressible Multi-Fluids Flows
}

\author{
H. W. Zheng* and N. Qin ${ }^{\dagger}$ \\ Department of Mechanical Engineering, University of Sheffield, Mappin Street, Sheffield S1 3JD, U.K \\ C. $\mathrm{Shu}^{\ddagger}$ \\ National University of Singapore, 10 Kent Ridge Crescent, Singapore 119260
}

In this paper, a hexahedral-mesh based solution adaptive algorithm for the simulation of compressible multi-fluid flows is proposed. The data structure, the refinement and coarsening process, and the solution adaptive method are described. The cells with different levels are stored in different lists. This avoids the recursive calculation of solution of mother (non-leaf) cells. Besides, the faces are separated stored into two lists: one for leaf faces and another for non-leaf faces. Thus, high efficiency is obtained due to these features. Numerical results show that there is no oscillation of pressure and velocity across the interface and it is feasible to apply it to solve compressible multi-fluid flows with large density ratio (1000) and strong shock wave interaction with the interface.

\section{Nomenclature}

$A_{f} \quad$ area of the face $f$

$E$ total energy

$\gamma \quad$ property of the material

$\mathbf{n}_{f} \quad$ normal direction of the face $f$

U conservative variable vector

$p$ pressure

$\Phi_{f} \quad$ numerical flux at the face $f$

$\pi \quad$ property of the material

$p^{* j} \quad$ intermediate pressures

$r \quad$ location of the initial interface

$r_{0} \quad$ initial radius

$\rho$ density

$\mathbf{R}$ residual vector

$q_{f} \quad$ HLLC intermediate normal velocity

$s_{j} \quad$ intermediate signal speeds

S source vector

$t$ physical time

u velocity vector

$u_{n}^{j} \quad$ normal velocity

$V_{c} \quad$ volume of cell c

\footnotetext{
*Research Associate, Email: H.Zheng@sheffield.ac.uk.

$\dagger$ Professor of aerodynamics, and AIAA Associate Fellow.

$\ddagger$ Professor
} 


\section{Introduction}

The compressible multi-fluid flows have been intensively investigated by many researchers. ${ }^{1-16}$ In general, most of them are three dimensional. Besides, due to complexity of these problems such as steep material interface, density and pressure gradients that occur in such flows, accurate predictions of flow structure of compressible multi-fluid flows pose a major challenge. Thus, it is necessary to develop the three dimensional (3D) adaptive mesh technique to study the problems.

However, most work on compressible multi-fluid flows employ 2D Cartesian based adaptive method. ${ }^{6-9}$ For example, ${ }^{6,8}$ presented the work by combining the level set method and the 2D Cartesian adaptive meshes. For this method, a complex modified coarse-to-fine and fine-to-coarse inter-level transfer operators need to be employed to stabilize the computation. Apart from Cartesian adaptive meshes, there is another paper ${ }^{9}$ which employs the 2D moving mesh method and real ghost fluid method. ${ }^{17}$ Their extension and applications to $3 \mathrm{D}$ compressible multi-fluid flows are still rarely conducted. In this paper, we extend the $2 \mathrm{D}$ quadrilateral based adaptive technique ${ }^{13}$ to $3 \mathrm{D}$ hexahedral based adaptive method.

The rest of the paper is organized as follows. In the second section, the governing equations are described. In the third section, the data structure, the criterion for refinement and coarsening and the solution adaptive method for compressible multi-fluid flows are presented. The numerical results are given in the fourth section.

\section{Governing equations}

The governing equations for the compressible multi-fluid flows with stiffened gas equation of state (EOS) are Euler equations

$$
\partial_{t} \int_{\Omega}\left(\begin{array}{c}
\rho \\
\rho \mathbf{u} \\
E
\end{array}\right) d V+\int_{\partial \Omega}\left(\begin{array}{c}
\rho \mathbf{u} \\
\rho \mathbf{u} \otimes \mathbf{u}+P[I] \\
(E+P) \mathbf{u}
\end{array}\right) \cdot \mathbf{n} d S=0,
$$

where $\rho$ is the density, $\mathbf{u}$ is the velocity, and $E$ is the total energy.

To tackle the well-known difficulty ${ }^{11,14}$ of spurious pressure oscillations at material interfaces, it requires to solve another two transport equations

$$
\frac{\partial \Xi}{\partial t}+\mathbf{u} \cdot \nabla \Xi=0
$$

with,

$$
\Xi=\left(\begin{array}{c}
\beta \\
\theta
\end{array}\right), \beta=\frac{1}{\gamma-1}, \theta=\frac{\gamma \pi}{\gamma-1}
$$

Here, $\beta$ and $\theta$ is a function of are the property of the material $(\gamma$ and $\pi) . \beta$ is also used to indicate the position of the interface. These two variables are also used to calculate the pressure $p$ according to stiffened gas EOS,

$$
p=\left(E-\frac{1}{2} \rho u^{2}+\theta\right) / \beta
$$

\section{Adaptive method}

The adaptive grid generator developed in this work belongs to the unstructured adaptive grid family. In the following, the data structure, local refinement, local coarsening and the solution adaptive procedure are described.

\section{A. Data structure}

The adaptive mesh consists of nodes, faces and cells. All nodes, faces and cells are stored in different dynamically allocated lists ${ }^{13}$ which are well-designed that the adding, deleting of a certain object is only of the order of $\mathrm{O}(1)$. For each cell, it stores the pointers (or indices) to the six faces and eight nodes (Fig. 1). Each face stores the pointers to the two neighboring cells. The pointer (or indices) to the four sub-faces is also included in face data structure. As a result, the adding or deleting node of a face does not need to access the neighboring cells. Hence, for each cell, there is no need to store the pointers (or indices) of the eight 
child cells as they can be accessed by using the sub-faces of the six faces. What is more, the neighboring cells of a cell can be obtained by visiting the six faces.

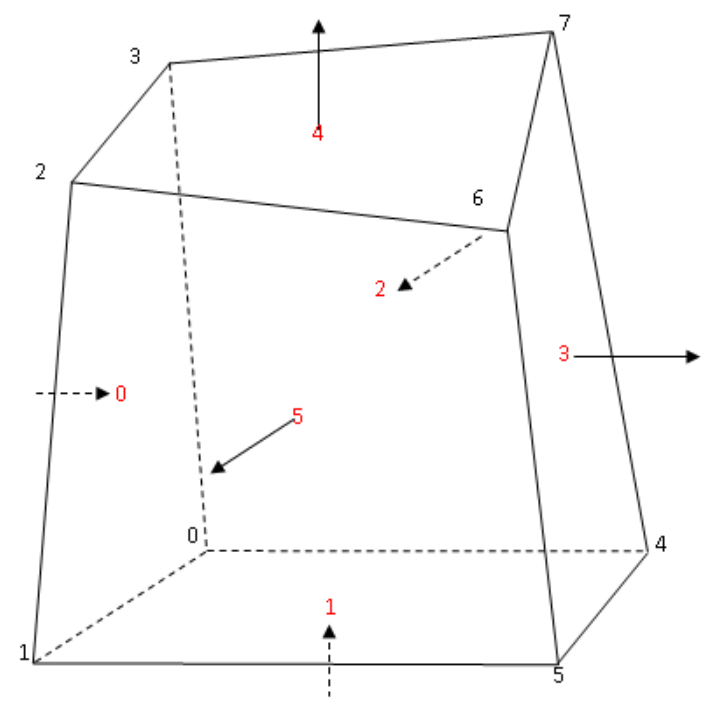

Figure 1. Data Structure

After each refinement of a cell, each cell is split into eight cells and each face of this cell is split into four sub-faces (Fig. 2). These cells are further organized into different lists according to their level and the faces are separated further stored into two lists: one for leaf faces and another for non-leaf faces. Specially care needs to be taken to reset the pointers to the new nodes of each face during the refinement (Fig. 3).

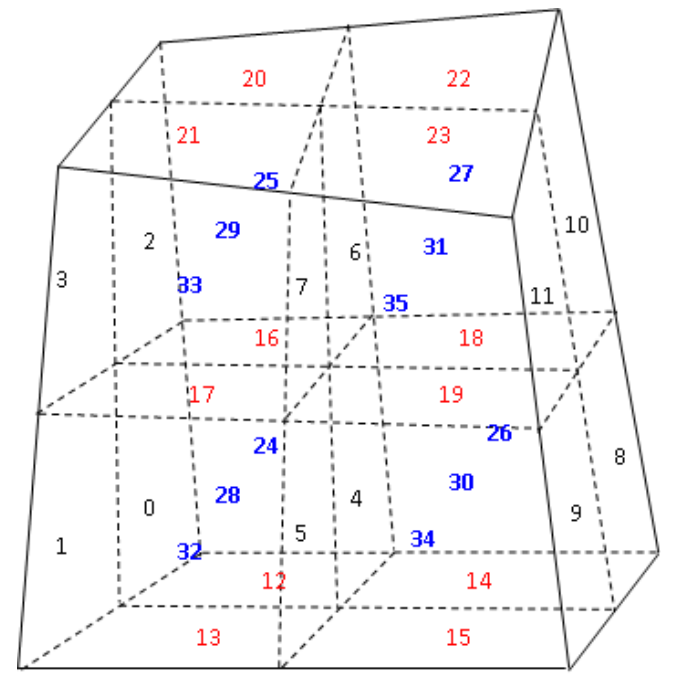

Figure 2. Faces created after refining a cell

\section{B. Adaptive criterion for refinement and coarsening}

In order to determine which cell is to be refined or be coarsened, the error detector is required. Here, the error detector is the maximum of the second-order Taylor truncation error over the first-order Taylor truncation 


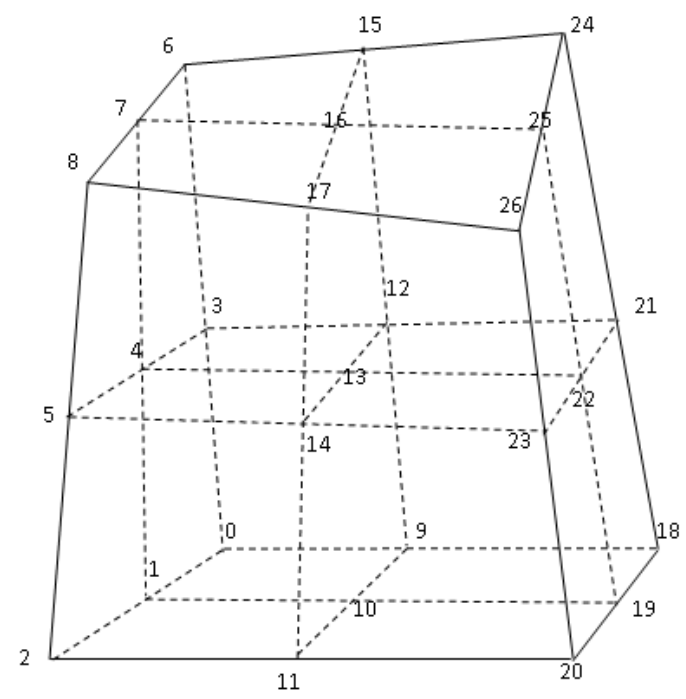

Figure 3. Nodes created after refining a cell

error of density of each face,

$$
\varepsilon_{T}=\max _{f}\left(\frac{\left|\left(\nabla_{l_{n}} \rho\right)_{C}-\left(\nabla_{l_{n}} \rho\right)_{L}\right|}{\alpha_{f} \rho_{C} / d l+\left|\left(\nabla_{l_{n}} \rho\right)_{L}\right|}, \frac{\left|\left(\nabla_{l_{n}} \rho\right)_{C}-\left(\nabla_{l_{n}} \rho\right)_{R}\right|}{\alpha_{f} \rho_{C} / d l+\left|\left(\nabla_{l_{n}} \rho\right)_{R}\right|}\right)_{f}
$$

If the value of error detector of a target cell is greater than the pre-defined refining threshold $\varepsilon_{R}$, then the cell needs to be refined; otherwise, if it is smaller than $\varepsilon_{C}$, then the cell needs to be coarsened.

However, there are still two more constraints for the refinement process. One is that the cell has no children (sub-cells). The other one is that the maximum difference of the levels between the current cell and all the neighboring cells cannot be greater than one. Similarly, there are two more constraints for coarsening. The first one is that the cell must have children (sub-cells). The second one is that the maximum difference of the levels between the current cell and all the neighboring cells cannot be greater than one.

\section{Solution adaptive method}

In this method, in order to avoid the recursive calculation, the cells are separately stored in different lists according to their levels. Thus, the solution can be obtained in a level by level manner so that the cells of high level are calculated after the cells of low level.

\section{Time evolution}

The set of equation Eq. (1) can be easily discretized at each leaf cell by the two stage Runge-Kutta schemes, ${ }^{13}$

$$
\mathbf{U}_{c}^{(*)}=\mathbf{U}_{c}^{n}-\alpha \operatorname{Res}\left(U_{c}^{n}\right)
$$

and

$$
\mathbf{U}_{c}^{n+1}=0.5\left[\mathbf{U}_{c}^{n}+\mathbf{U}_{c}^{(*)}-\alpha \mathbf{R}_{c}\left(\mathbf{U}_{c}^{(*)}\right)\right]
$$

where $U$ is the state vector $\left(\begin{array}{ccccc}\rho & \rho \vec{u} & E & \beta & \theta\end{array}\right)^{T}, \Delta t$ is the time step, $V_{c}$ is the volume of cell $c, \mathbf{R}_{c}$ is the residual, and $\alpha$ is the ratio between time step and the volume $\left(\alpha=\Delta t / V_{c}\right)$.

\section{Residual calculation}

The face-based technique is employed to calculate the residual. That is, the residual $\left(\mathbf{R}_{f 1 \rightarrow L}\right)$ at the left neighboring cell center $(f 1 \rightarrow L)$ of a leaf face $f 1$ is updated in the following way,

$$
\mathbf{R}_{f 1 \rightarrow L}=\mathrm{R}_{f 1 \rightarrow L}+\Phi_{f 1}\left(\mathbf{U}^{-}, \mathbf{U}^{+}, \mathbf{n}_{f 1}\right) \cdot A_{f 1}
$$


and the residual $\left(\mathbf{R}_{f 1 \rightarrow R}\right)$ at the right cell center $(f 1 \rightarrow R)$ of this leaf face $f 1$ is updated by

$$
\mathbf{R}_{f 1 \rightarrow R}=\mathbf{R}_{f 1 \rightarrow R}-\Phi_{f 1}\left(\mathbf{U}^{-}, \mathbf{U}^{+}, \mathbf{n}_{f 1}\right) \cdot A_{f 1}
$$

In order to achieve the object of the spurious pressure oscillations-free material interfaces under complex geometry, the solving of the last two sub-equations in Eq. (1) are important. In this paper, we adopt a HLLC consistent way in paper, ${ }^{13}$ the HLLC scheme is embedded by updating the last two items of residue as

$$
\mathbf{R}_{\Xi, c}=\mathbf{R}_{\Xi, c}-\Xi_{c} \sum_{f} q_{f} \cdot A_{f}
$$

Here, $q_{f}$ is the HLLC intermediate normal velocity ${ }^{13}$ at the face.

\section{Flux calculation}

To discretize the convection term, the Harten, Lax and van Leer approximate Riemann solver with the Contact wave restored (HLLC) numerical flux for an face $f$ is adopted for compressible multi-fluid flows, ${ }^{13}$

$$
\Phi_{f}\left(U^{-}, U^{+}, \mathbf{n}\right)=\frac{1}{2}\left[\Phi^{-}+\Phi^{+}-\operatorname{sign}\left(s_{\mathrm{m}}\right)\left(\mathbf{U}^{+}-\mathbf{U}^{-}\right)\right]
$$

with

$$
\Phi^{j}=\mathbf{F}\left(\mathbf{U}^{j}\right) \cdot \mathbf{n}+s_{j}^{\#}\left(\mathbf{U}^{* j}-\mathbf{U}^{j}\right), j=-,+
$$

where the intermediate states can be calculated by

$$
\mathbf{U}^{* j}=\frac{s_{j}-u_{n}^{j}}{s_{j}-s_{m}}\left(\begin{array}{c}
\rho^{j} \\
\rho^{j}\left[\mathbf{u}^{j}-\left(u_{n}^{j}-s_{m}\right) \mathbf{n}\right] \\
E^{j} \\
\Xi^{j}
\end{array}\right)+\left(\begin{array}{c}
0 \\
0 \\
\left(p^{* j} s_{m}-p^{j} u_{n}^{j}\right) /\left(s_{j}-u_{n}^{j}\right) \\
0
\end{array}\right), j=-,+
$$

with

$$
p^{* j}=\rho^{j}\left(u_{n}^{j}-s_{j}\right)\left(u_{n}^{j}-s_{m}\right)+p^{j}, j=-,+
$$

Here, $s_{j}$ and $p^{* j}$ are the intermediate signal speeds and pressures of HLLC scheme. ${ }^{13}$

\section{Results and Discussion}

In order to validate the performance of the present three-dimensional solution adaptive technique for compressible multi-fluid flows, test cases of material interface problem and bubble interaction with shock wave are considered herein.

\section{Interface translation problem}

The interface translation problem is used to demonstrate the oscillation-free feature of current solver. Initially, one fluid with a spherical shape surrounded by another fluid is put at the position $(0.25 \mathrm{~m}, 0.25 \mathrm{~m}, 0.25 \mathrm{~m})$ of the domain. The radius of the spherical interface is $r_{0}=0.16 \mathrm{~m}$. The periodic boundary condition is employed at all boundaries. The initial mesh is adaptively generated by a $20 \times 20 \times 20$ background mesh (level

0 ) with the finest resolution level as 4 . The initial values of the primitive variables for the two fluids are given by, ${ }^{11}$

$$
\begin{aligned}
& \rho_{1}=0.1 \mathrm{~kg} / \mathrm{m}^{3}, u_{1}=1 \mathrm{~m} / \mathrm{s}, v_{1}=1 \mathrm{~m} / \mathrm{s}, w_{1}=1 \mathrm{~m} / \mathrm{s}, p_{1}=1 P a, \gamma_{1}=1.4, \pi_{1}=0 P a, \text { forr }<r_{0} \\
& \rho_{2}=1 \mathrm{~kg} / \mathrm{m}^{3}, u_{2}=1 \mathrm{~m} / \mathrm{s}, v_{2}=1 \mathrm{~m} / \mathrm{s}, w_{2}=1 \mathrm{~m} / \mathrm{s}, p_{2}=1 P a, \gamma_{2}=1.6, \pi_{2}=0 P a, \text { forr }>=r_{0}
\end{aligned}
$$

This initial condition indicates that there are no shock and other perturbation in the flow field. As a result, the inner fluid with spherical shape should move with the constant velocity along the diagonal direction. 
It is used to verify the solver and see whether there is no oscillation of pressure and velocity across the interface.

We plot the interface position at $t=0$ second and $t=0.18$ second as shown in Fig. 4 and Fig. 5 . As stated above, the spherical interface moves with a constant velocity. Hence, there is an analytical solution for the interface position. From Fig. 4, it can be easily observed that the calculated position and shape of the interface at $\mathrm{t}=0.18$ second agrees well with the predicted one.

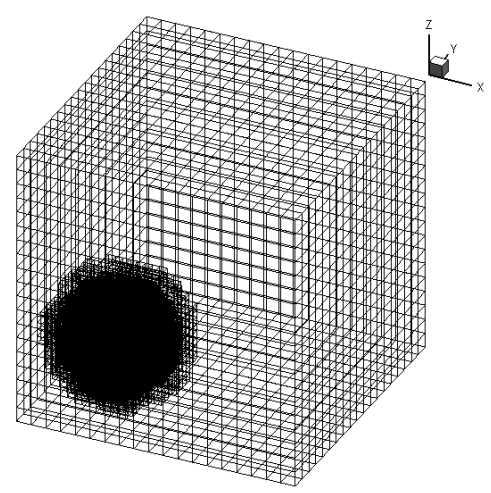

(a)

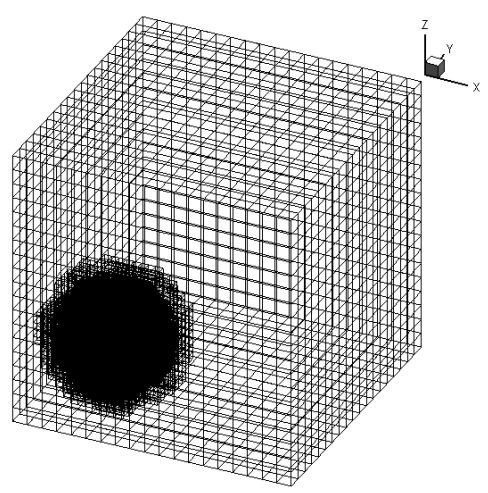

(b)

Figure 4. Mesh (a) and density iso-surface (b) at time $t=0$ s

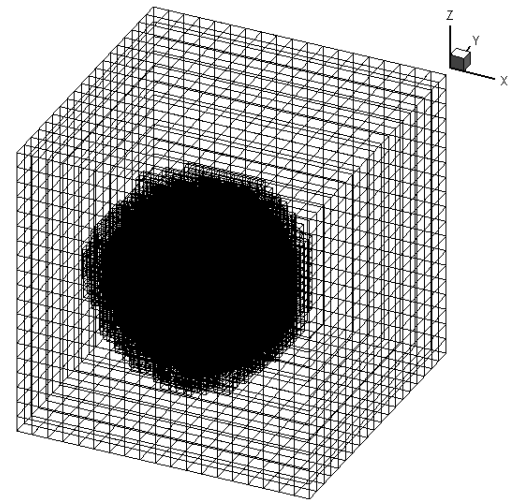

(a)

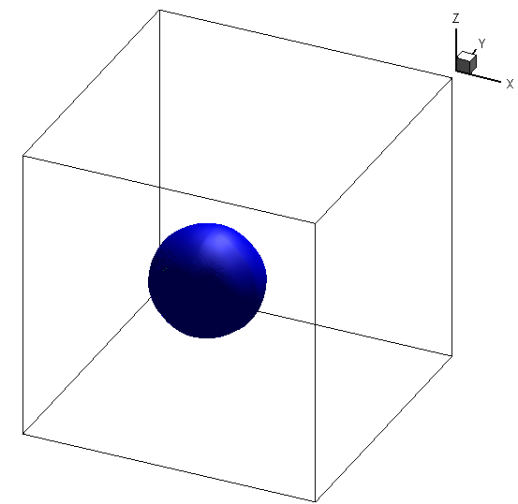

(b)

Figure 5. Mesh (a) and density iso-surface (b) at time $t=0.18 \mathrm{~s}$

Besides, the computed pressure and velocity are plotted in Fig. 6. It is clearly the pressure and velocity is uniform. These shows that they do not oscillate around the interface which confirms the claim of oscillationfree of our adaptive method.

\section{Bubble-shock interaction}

In this section, the bubble shock interaction with a large density ratio (1000) and pressure ratio between the water and gas bubble is investigated. Initially, a left going planar shock wave (Fig. 7) with Mach number of 1.422 traveling in the water and a circular bubble gas with radius of $0.2 \mathrm{~m}$ are put at the left side $(0.7 \mathrm{~m}$, $0.5 \mathrm{~m}, 0.5 \mathrm{~m})$ of the shock wave. The domain is $[0,1.2] \times[0,1] \times[0,1] \mathrm{m}^{3}$ and the shock wave is located at $0.95 \mathrm{~m}$.

The pre-shock and post-shock fluids are initially at rest and the parameters such as density, velocity, pressure and the material parameters are listed as follows, ${ }^{15}, 16$

$$
\rho_{1}=1000 \mathrm{~kg} / \mathrm{m}^{3}, u_{1}=0 \mathrm{~m} / \mathrm{s}, v_{1}=0 \mathrm{~m} / \mathrm{s}, w_{1}=0 \mathrm{~m} / \mathrm{s}, p_{1}=1 \cdot 10^{5} \mathrm{~Pa}, \gamma_{1}=4.4, \pi_{1}=6 \cdot 10^{8} \mathrm{~Pa}
$$




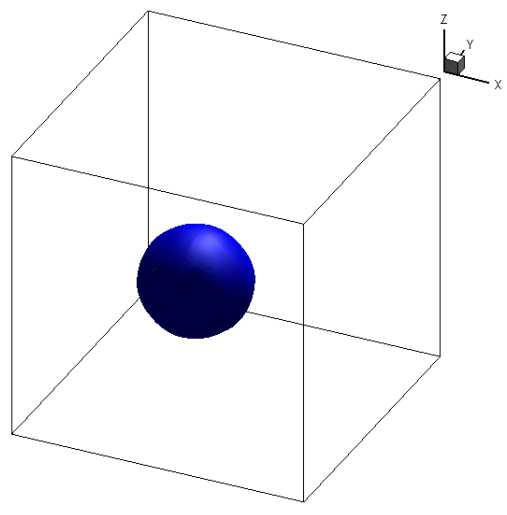

(a)

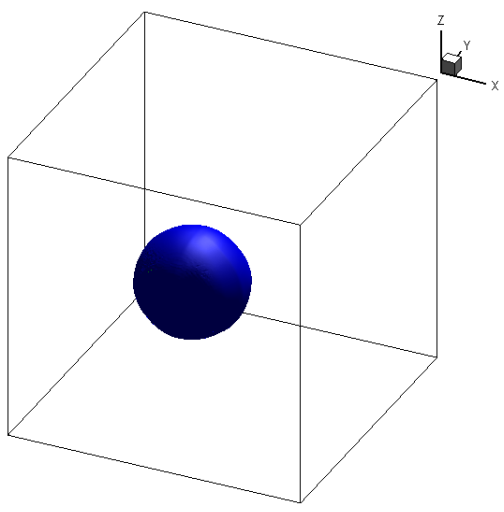

(b)

Figure 6. Slice of velocity $(u)$ contour (a) and pressure contour (b) at time $t=0.18 \mathrm{~s}$

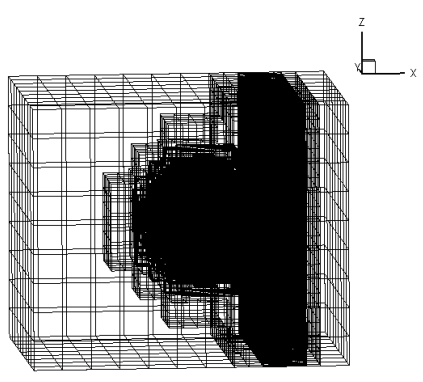

(a)

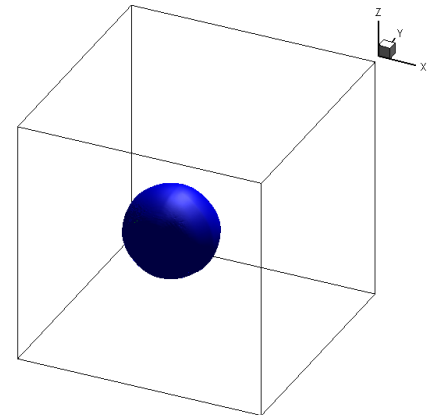

(b)

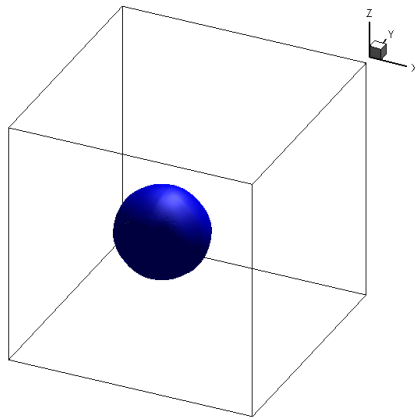

(c)

Figure 7. Mesh (a), density iso-surface (b) and interface iso-surface (c) at time $t=0$ s

$$
\rho_{3}=1230 \mathrm{~kg} / \mathrm{m}^{3}, u_{3}=-432.69 \mathrm{~m} / \mathrm{s}, v_{3}=0 \mathrm{~m} / \mathrm{s}, w_{3}=0 \mathrm{~m} / \mathrm{s}, p_{3}=10^{9} \mathrm{~Pa}, \gamma_{3}=4.4, \pi_{3}=6 \cdot 10^{8} \mathrm{~Pa}
$$

The primitive variables for the bubble are set as,

$$
\rho_{2}=1.2 \mathrm{~kg} / \mathrm{m}^{3}, u_{2}=0 \mathrm{~m} / \mathrm{s}, v_{2}=0 \mathrm{~m} / \mathrm{s}, v_{2}=0 \mathrm{~m} / \mathrm{s}, p_{2}=10^{5} \mathrm{~Pa}, \gamma_{2}=1.4, \pi_{2}=0.0 P a
$$

The reflective boundary conditions are employed at the top and bottom boundary, while the extrapolation boundary conditions are imposed at the left and right boundary. This is a challenging problem due to the large pressure jump across the shock wave and the large ratio of the acoustic impedances of the liquid to gas.

The simulation is performed on the adaptive mesh generated by a uniform background mesh (level is 0 ) of $12 \times 10 \times 10$ with the finest resolution level as 4 . The mesh and the iso-surface of density and $\beta$ at different time from 0s to $0.0005 \mathrm{~s}$ are plotted in Fig. $7-12$.

It can be easily observed that the adaptive mesh can reflect the main flow features such as shock wave, and material interface etc. As can be seen in Fig. 9, the shock wave first propagates in the water. After the shock wave hits the bubble, a circular wave is generated and reflected from the interface (Fig. 10-(c)). Then the left-going shock wave continues to propagate through the bubble and the reflected circular wave moves outward as shown in Fig. 11. When the right moving circular wave hits the upper and lower boundary, the second reflected wave is generated. The second reflected wave will interact with other waves to form a complex flow (Fig. 12). 


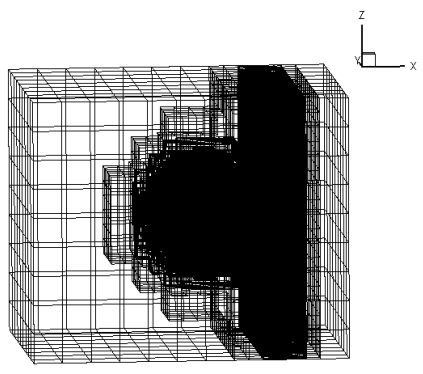

(a)

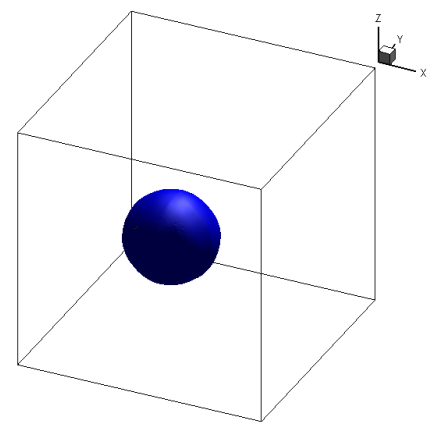

(b)

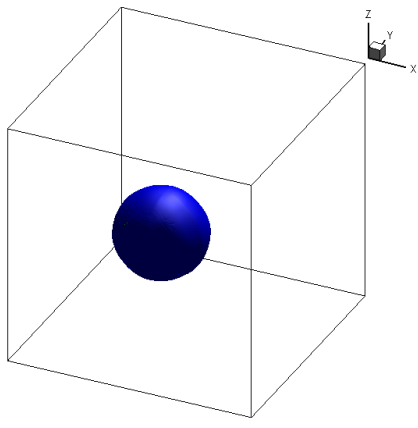

(c)

Figure 8. Mesh (a), density iso-surface (b) and interface iso-surface (c) at time $t=1 \mathrm{e}-4 \mathrm{~s}$

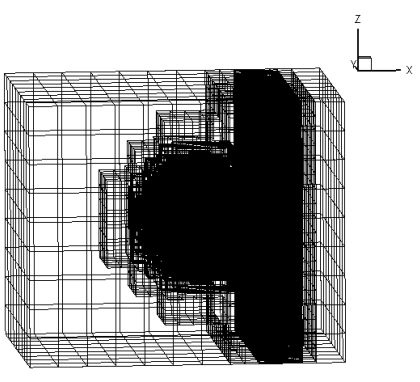

(a)

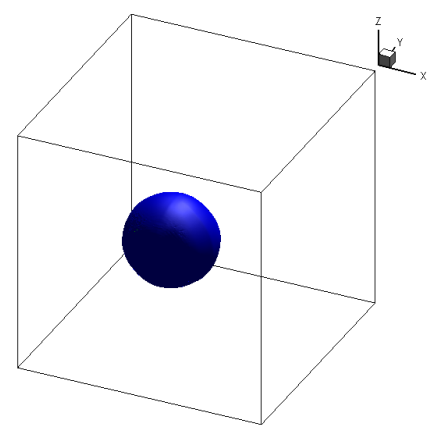

(b)

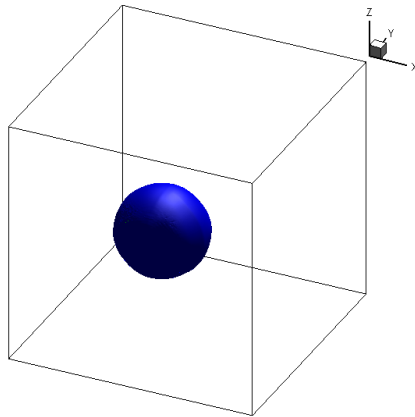

(c)

Figure 9. Mesh (a), density iso-surface (b) and interface iso-surface (c) at time $t=2 e-4 s$

\section{Conclusion}

In this paper, a hexahedral based solution adaptive method for 3D compressible multi-fluid flows developed is proposed. The approach is able to automatically and efficiently adjust the local mesh density to reflect the transient behavior of the solution. Two examples have been carried out to examine the performance of the present solver for compressible multi-fluid flows. They show the oscillation-free of pressure and velocity across the interface and it is feasible in solving compressible multi-fluid flows with large density ratio (1000) and strong shock wave (the pressure ratio is 10000) interaction with the interface.

\section{References}

${ }^{1}$ Larouturou, B., How to preserve the mass fraction positive when computing compressible multi-component flows, J. Comput. Phys., 95, 59-84, 1991.

${ }^{2}$ Abgrall, R., and Karni, S., Computations of Compressible Multifluids, J. Comput. Phys., 169, 594-623, 2001.

${ }^{3}$ Shyue, K.M., An efficient shock-capturing algorithm for compressible multi-component problems, J. Comput. Phys., 142, 208-242, 1998

${ }^{4}$ Shyue, K.M., A fluid-mixture type algorithm for barotropic two-fluid flow problems, J. Comput. Phys. 200, 718-748, 2004.

${ }^{5}$ Johnsen, E. and Colonius, T., Implementation of WENO schemes for compressible multicomponent flow problems, J. Comput. Phys., 219, 715-732, 2006.

${ }^{6}$ Nourgaliev, R.R., Dinh, T.N., and Theofanous, T.G., Adaptive Characteristics-Based Matching for Compressible Multifluid Dynamics, J. Comput. Phys., 213, 500-529, 2006.

${ }^{7}$ Banks, J.W., Schwendeman, D.W., Kapila, A.K., and Henshaw W.D., A high-resolution Godunov method for compressible multi-material flow on overlapping grids, J. Comput. Phys., 223, 262-297, 2007.

${ }^{8}$ Kadioglu, S.Y. and Sussman, M., Adaptive solution techniques for simulating underwater explosions and implosions, J. Comput. Phys., 227, 2083-2104, 2008. 


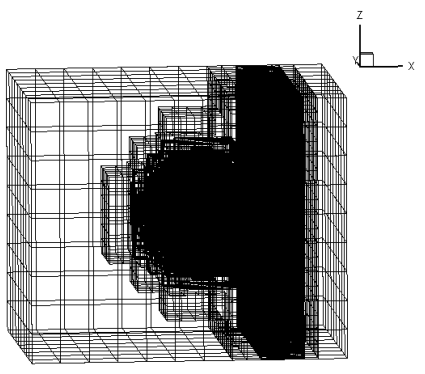

(a)

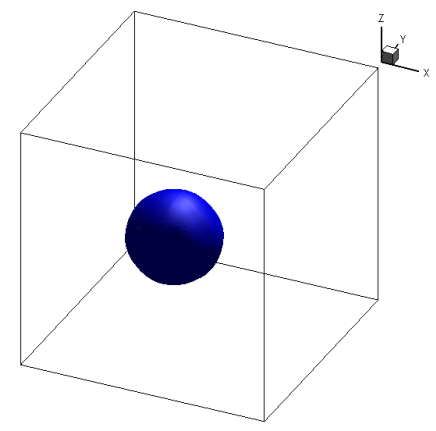

(b)

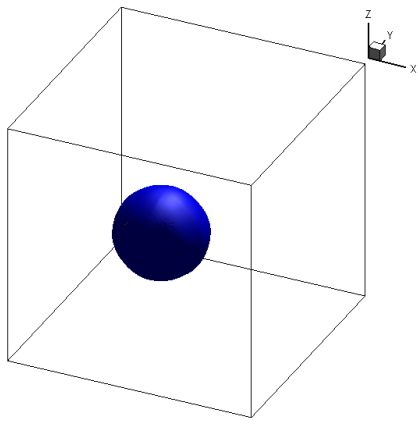

(c)

Figure 10. Mesh (a), density iso-surface (b) and interface iso-surface (c) at time $t=3 e-4 s$

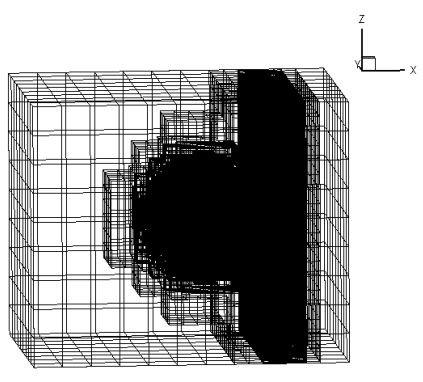

(a)

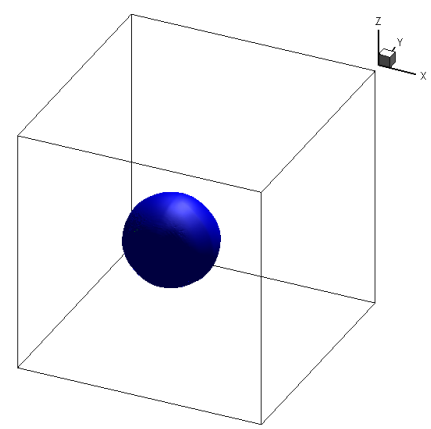

(b)

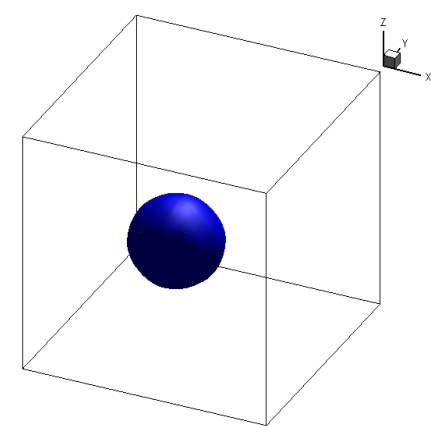

(c)

Figure 11. Mesh (a), density iso-surface (b) and interface iso-surface (c) at time $t=4 e-4 s$

${ }^{9}$ Wang, C., Tang H., and Liu, T.G., An adaptive ghost fluid finite volume method for compressible gas-water simulations, J. Comput. Phys., 227, 6385-6409, 2008.

${ }^{10}$ Saurel, R., Gavrilyuk, S., and Renaud F., A multiphase model with internal degrees of freedom: application to shockbubble interaction, JFM, 495, 283-321, 2003.

${ }^{11}$ Shyue, K.M., An efficient shock-capturing algorithm for compressible multi-component problems, J. Comput. Phys., 142, 208-242, 1998.

${ }^{12}$ Zhang, Q, Graham, M.J., A numerical study of Richtmyer-Meshkov instability driven by cylindrical shocks, Phys. Fluids, 10, 974-92, 1998.

${ }^{13}$ Zheng, H. W., Shu, C. and Chew, Y.T., An Object-Oriented and Quadrilateral-mesh based Solution Adaptive Algorithm for Compressible Multi-fluid Flows, J. Comput. Phys., 227, 6895-6921, 2008.

${ }^{14}$ Abgrall, R., and Karni, S., Computations of Compressible Multifluids, J. Comput. Phys. 169, 594-623, 2001.

${ }^{15}$ Allaire, G, Clerc, S, and Kokh, S., A five-equation model for the simulation of interfaces between compressible fluids, J. Comput. Phys., 181, 577-616, 2002.

${ }^{16}$ Shyue, K.M., A fluid-mixture type algorithm for compressible multicomponent flow with van der Waals equation of state, J. Comput. Phys., 156, 43-88, 1999.

${ }^{17}$ Wang, C.W., T.G. Liu, B.C. Khoo, A real-ghost fluid method for the simulation of multi-medium compressible flow, SIAM J. Sci. Comput., 28, 278-302, 2006. 


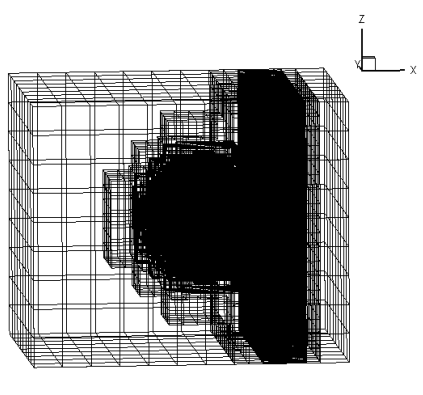

(a)

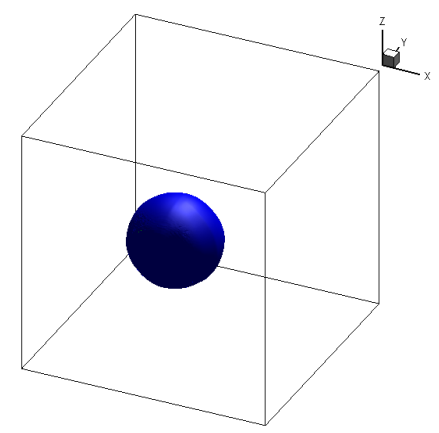

(b)

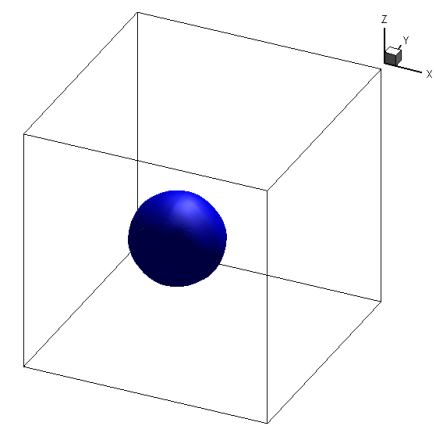

(c)

Figure 12. Mesh (a), density iso-surface (b) and interface iso-surface (c) at time $t=5 e-4 s$ 